\title{
Silk-like secretion from tarantula feet
}

\section{An unsuspected attachment mechanism may help these huge spiders to avoid catastrophic falls.}

Spiders spin silk from specialized structures known as abdominal spinnerets - a defining feature of the creatures ${ }^{1,2}-$ and this is deployed to capture prey, protect themselves, reproduce and disperse. Here we show that zebra tarantulas (Aphonopelma seemanni) from Costa Rica also secrete silk from their feet to provide adhesion during locomotion, enabling these spiders to cling to smooth vertical surfaces. Our discovery that silk is produced by the feet provides a new perspective on the origin and diversification of spider silk.

Spider feet have a 'dry' attachment system that relies on van der Waals forces generated by thousands of spatulate hairs ${ }^{3}$. Additionally, small distal claws enhance adhesion to rough surfaces by mechanically interlocking with the substrate. We have discovered that the tarantula A. seemanni (Fig. 1) has a third attachment mechanism, which depends on fibres exuded from nozzle-like structures on its feet. These fibrous secretions (Fig. 2) function as silken tethers and, when laid down on glass plates, appear as 'footprints' that consist of dozens of fibres with diameters of 0.2-1.0 micrometres and lengths of 100-2,500 micrometres (for details of analyses, see supplementary information).

Individual tarsal silk fibres often start as a flattened plaque. This seems to be secreted as a viscous fluid that solidifies, gluing the thread to the substrate. The function and morphology of the tarantula's tarsal silk resembles the adhesive agent produced by the spinnerets of the spider Antrodiaetus unicolor ${ }^{4}$, as well as the attachment (pyriform) silk that many spiders use to cement their draglines to substrates ${ }^{2}$.

We induced $A$. seemanni to walk on vertical glass surfaces in order to observe the contact mechanics of this challenging locomotion. When walking up vertical planes, the spider attached only the distal parts of its tarsi to the substrate. As it started to slip down the glass, silk produced by the tarsal spigots on all four pairs of legs arrested the spider's descent and allowed it to remain attached to the vertical surface. The spider's feet were positioned such that the silk-producing spigots were in contact with the glass, while the dense setae in adjacent regions were held off the surface.

Our discovery of secreted tarsal silk forces a reconsideration of the evolution of spider silks ${ }^{1,5,6}$. Depending on its distribution across spider phylogeny, tarsal synthesis of silk could represent the ancestral condition, with silk

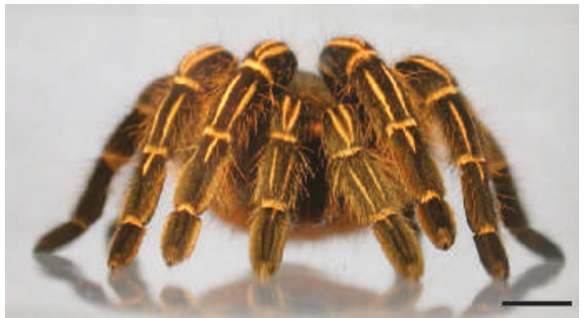

Figure 1 Costa Rican zebra tarantula,

Aphonopelma seemanni. Female, anterior view. Scale bar, $5 \mathrm{~mm}$.

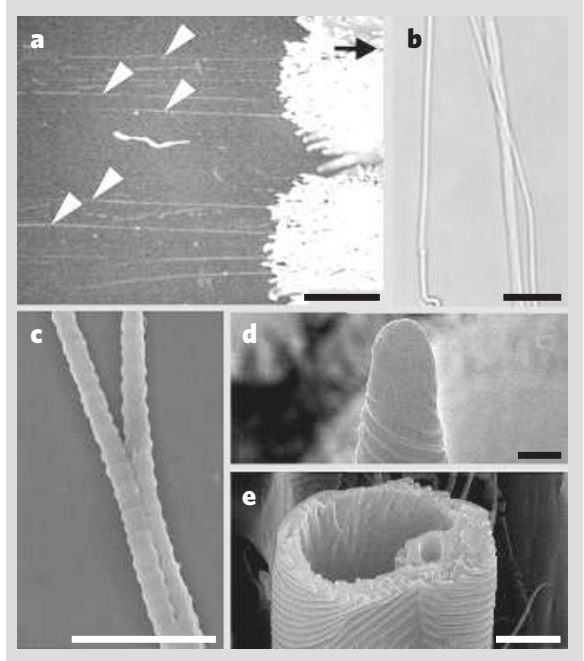

Figure 2 | Tarsal silk production by the spider Aphonopelma seemanni. a, Fibres left behind (arrowheads) by a spider sliding down a vertical glass surface. Black arrow indicates direction of sliding. The spherical structures are the distal part of the tarsus (scopula), covered with hairs and spigots. $b$, Traces left by the tarsus of a spider walking on a coverslip. c, Single fibres observed by cryoscanning electron microscopy. $d$, Tip of a tarsal spigot with the opening obstructed by silk. e, Tarsal spigot broken near the base. Scale bars: a, $500 \mu \mathrm{m}$; b, $10 \mu \mathrm{m}$;, $1 \mu \mathrm{m}$; d, $2 \mu \mathrm{m}$; e, $5 \mu \mathrm{m}$.

production from abdominal spinnerets evolving later. Alternatively, because the tarantula clade (Theraphosidae) is a species-rich group that includes the largest known spiders ${ }^{7}$, tarsal production of silk may have evolved independently as a key innovation to enhance locomotor ability and avert catastrophic falls.

Both evolutionary hypotheses are consistent with the homology of legs and spinnerets as arthropod appendages ${ }^{1,5,8}$. Regardless of whether tarsal silk production is ancestral or secondarily derived, the silk-producing apparatus of spiders seems to be controlled by developmental modules that can be expressed in a variety of body parts.

Investigation of the genes involved in tarsal silk production should resolve whether the original function of spider silk was to increase traction or whether it was later coopted for that purpose. Spinneret silk proteins are encoded by a gene family that has evolved through a series of gene duplications and subsequent modifications for particular tasks ${ }^{9-11}$. If tarsal silks belong to the same gene family, then comparison of tarsal and spinneret silks should help our understanding of the ancestral function and composition of spider silk.

Stanislav N. Gorb ${ }^{\star} \uparrow$, Senta Niederegger ${ }^{\star} \uparrow+$ Cheryl Y. Hayashi\$, Adam P. Summers|l, Walter Vötsch $\uparrow$, Paul Waltherף

*Evolutionary Biomaterials Group, Max Planck Institute for Metals Research, 70569 Stuttgart,

Germany

e-mail:s.gorb@mf.mpg.de

$\uparrow$ Max Planck Institute for Developmental Biology, 71076 Tübingen, Germany

Institute of Forensic Medicine, Friedrich Schiller University of Jena, 07743 Jena, Germany §Department of Biology, University of California, Riverside, California 92521, USA

||Ecology and Evolutionary Biology, University of California, Irvine, California 92697, USA

IElectron Microscopy Department, University of Ulm, 89069 Ulm, Germany

1. Shultz, J. W. Biol. Rev. 62, 89-113 (1987).

2. Vollrath, F. \& Knight, D. P. Nature 410, 541-548 (2001).

3. Arzt, E., Gorb, S. \& Spolenak, R. Proc. Natl Acad. Sci. USA 100, $10603-10606$ (2003).

4. Palmer, J. M., Coyle, F. A. \& Harrison, F. W. J. Morphol. 174, 269-274 (1982).

5. Bristowe, W. S. Proc. Zool. Soc. Lond. 103, 1015-1057 (1932).

6. Craig, C. L. Annu. Rev. Entomol. 42, 231-267 (1997).

7. Coddington, J. A. \& Levi, H. W. Annu. Rev. Ecol. Syst. 22, 565-592 (1991).

8. Damen, W. G. M., Saridaki, T. \& Averof, M. Curr. Biol. 12, 1711-1716 (2002)

9. Guerette, P. A., Ginzinger, D. G., Weber, B. H. F. \& Gosline, J. M. Science 272, $112-115$ (1996)

10. Gatesy, J., Hayashi, C., Motriuk, D., Woods, J. \& Lewis, R. Science 291, 2603-2605 (2001).

11. Garb, J. E., DiMauro, T., Vo, V. \& Hayashi, C. Y. Science 312, 1762 (2006)

Supplementary information accompanies this communication on Nature's website.

Received 3 July; accepted 25 August 2006. Competing financial interests: declared none. doi:10.1038/443407a

BRIEF COMMUNICATIONS ARISING online www.nature.com/bca see Nature contents. 